\title{
Développement régional : l'échec démocratique de la régionalisation
}

\author{
Donald Martel et Jean-François Albert ${ }^{1}$ \\ MRC de Nicolet-Yamaska
}

\section{Préambule}

Le métier d'administrateur municipal exige une discrétion quand il est question de prendre part à des débats publics sur la question municipale. Nous avons la chance d'influencer au quotidien le travail des élus, mais nous n'avons pas souvent l'occasion d'exprimer notre point de vue sur des questions aussi primordiales à nos yeux que le développement du milieu rural. Nous voulons remercier la revue Organisations et territoires de nous offrir cette plate-forme pour exprimer notre opinion sur l'occupation du territoire en milieu rural. Les propos dans le présent article sont teintés par notre expérience au sein de la MRC, mais n'engagent évidemment que notre opinion de professionnels et ne sauraient en rien représenter l'opinion des élus pour qui nous travaillons quotidiennement. Nous considérons travailler pour une des MRC les plus fonctionnelles du Québec, ce qui nous permet en quelque sorte d'être sereins avec les éléments de criti-
Le monde rural ne doit pas voir son salut dans sa croissance et dans l'atteinte d'un statut urbain. monologue sur un vibrant plaidoyer en faveur de la ruralité. Pour lui, la campagne est un milieu de vie intéressant et favorable à l'épanouissement des individus; pour lui, le monde rural ne souffre d'aucune comparaison avec la ville. Il s'agit d'une vision à laquelle nous souscrivons entièrement.

Le monde rural ne doit pas voir son salut dans sa croissance et dans l'atteinte d'un statut urbain. Au contraire, le monde rural devrait miser sur ses spécificités propres afin de générer du développement et d'assurer sa pérennité. Les élus ruraux ne devraient pas se fixer des objectifs de croissance dans l'intention de gonfler la population pour assurer la survie des municipalités. Ils devraient plutôt miser sur un réel développement durable qui suppose, entre autres, un milieu de vie attrayant, une économie vigoureuse et un environnement sain. Admettons-le d'emblée, le monde rural ne peut offrir tous les avantages de la ville (activités culturelles nombreuses, services variés, infrastructures de loique du présent article. No-

tre point de vue est celui de personnes ayant décidé de mener une vie dans le milieu rural et d'y travailler pour en faire avancer la cause. Nous souhaitons établir différents constats sur la ruralité d'un angle administratif, mais surtout dégager des pistes de solutions afin d'améliorer la destinée des régions rurales. Il s'agit d'un discours de praticiens qui vivent la ruralité d'un point de vue de gestionnaires d'une Municipalité régionale de comté.

\section{Ville et campagne : deux réalités complémentaires}

Dans le spectacle qu'il a présenté tout l'été dans sa petite municipalité rurale de Saint-Élie-de-Caxton, le désormais célèbre conteur Fred Pellerin termine son sir de prestige, etc.). Seule, une petite municipalité ne peut aspirer à offrir à ses citoyens des services à la hauteur de ceux qu'on retrouve en ville. C'est une des raisons pour lesquelles le monde rural doit travailler en complémentarité avec les milieux urbains qui l'entourent et développer en parallèle une réelle complicité entre les municipalités rurales afin d'offrir aux citoyens ruraux un milieu de vie intéressant. Les municipalités sont trop souvent en compétition entre elles : offre de terrains à rabais, congé de taxes foncières, joutes politiques pour l'obtention de subventions gouvernementales, etc. Par ailleurs, nous ne pouvons pas blâmer les élus locaux quant à leur intention de maintenir à tout prix leur niveau de population dans le contexte de décroissance démographique que nous observons dans plusieurs milieux. C'est une des raisons pour lesquelles il faut revoir la gouvernance en 
milieu rural. Il en sera question dans la section suivante.

Une grande difficulté qui freine le développement des milieux ruraux est la façon dont les municipalités tirent leurs revenus, soit le système de taxation sur la base du rôle foncier. Au moins trois éléments rendent cette façon de faire relativement inconciliable au développement des milieux ruraux. Pour prospérer et préserver les acquis, en cherchant généralement à ne pas hausser le compte de taxes de leurs citoyens, les élus municipaux ont comme obligation d'orienter leurs efforts en vue d'accroître la richesse foncière de leur municipalité. Dans des milieux en croissance qui n'attirent pas spontanément des investissements, les municipalités font souvent la promotion du développement résidentiel par des incitatifs fiscaux ou encore investissent des montants importants dans la promotion et le développement industriel, pour ne citer que ces deux exemples. Le plus souvent, ces actions sont posées en concurrence avec les municipalités voisines et à l'encontre de la protection de la zone agricole. Ainsi, à l'échelle régionale, des deniers publics sont dépensés afin que des municipalités obtiennent chacune une part du même gâteau, ce qui affaiblit nécessairement le monde municipal. Deuxièmement, puisque la MRC perçoit ses revenus en vertu d'un système de quote-part établi au prorata de la richesse foncière uniformisée, les municipalités n'ont pas intérêt à accroître leur participation financière dans la MRC, ce qui réduit la possibilité de celle-ci à contribuer efficacement au développement de son territoire. Finalement, le système actuel crée une iniquité entre les municipalités qui bénéficient d'une bonne situation géographique ou autres avantages stratégiques et les autres municipalités moins choyées quant à l'opportunité de tirer profit d'une plus avantageuse assiette fiscale.

\section{Le rôle des élus municipaux est essentiel pour assurer un sain développement du monde rural.}

Comme nous l'avons indiqué précédemment, les municipalités voient généralement leur salut dans la croissance de leur zone urbaine aux fins d'augmenter la richesse foncière. Pourtant, en milieu rural, une des façons d'occuper efficacement le territoire et d'assurer un développement durable est certainement de faire en sorte que le milieu profite au maximum des retombées de l'exploitation de ses ressources naturelles. À notre avis, il serait souhaitable que des mesures incitatives importantes soient fournies aux entrepreneurs qui décident de transformer les ressources naturelles à proximité d'où elles proviennent. Il serait aussi souhaitable, pour le bien collectif québécois, que le milieu rural perçoive des redevances financières ou divers avantages pour sa contribution à la protection de la zone agricole et aux ressources naturelles du territoire. Alors que le monde rural est souvent un mauvais protecteur de la zone agricole, il faudrait au contraire que les conditions le poussent à protéger cette zone d'importance stratégique au plan national.

\section{Le modèle d'organisation municipal}

Même si, en apparence, le modèle d'organisation municipal semble peu déterminant quant à l'avenir de l'occupation du territoire en milieu rural, nous pensons qu'il joue au contraire un rôle fondamental que nous aurions tort d'ignorer. À notre avis, le rôle des élus municipaux est essentiel pour assurer un sain développement du monde rural, et nous prétendons que la structure actuelle de la démocratie municipale ne favorise pas de façon optimale l'épanouissement des régions.

Pour que le monde municipal participe efficacement au développement du territoire, il faut favoriser une complicité entre les acteurs municipaux. Afin d'assurer une meilleure cohésion entre les petites municipalités (gérées par le Code municipal) et les municipalités rurales de plus grande importance (celles-là étant gérées par la Loi sur les cités et villes), le gouvernement créa les MRC en 1979 par l'adoption de la Loi sur l'aménagement et l'urbanisme. Les objectifs poursuivis étaient louables, mais nous constatons un échec quant à la création d'une réelle vision régionale de développement et une mise en commun des énergies propres à la création de richesse. Alors que les petites communautés devraient, selon nous, gérer dans un esprit collectif et de façon innovatrice, la structure municipale ne favorise pas l'atteinte de ces objectifs. Nous entendons par «structure municipale » les trois paliers de gouvernements municipaux, soit la Municipalité, la Municipalité régionale de comté et la Conférence régionale des élus.

Un de ces échecs consiste dans le mariage difficile entre les municipalités dites "centres » et les petites municipalités. Possédant souvent le pouvoir en raison 
de leur poids démocratique au Conseil des maires, les villes centres se positionnent souvent contre le renforcement des MRC au détriment d'une réelle symbiose entre les communautés d'un même milieu rural. Alors qu'elles devraient justement y trouver le cadre idéal à leur épanouissement, les petites municipalités se butent plutôt à des municipalités fortes qui ne favorisent pas leur développement. Il faut reconnaître que les villes centres ont généralement moins besoin des MRC en raison des ressources dont elles disposent. Le déséquilibre politique ainsi créé autour de la table de décision ne favorise pas le développement du territoire, la ville centre cherchant à profiter de ses infrastructures existantes et à en maximiser les retombées plutôt que de participer au développement d'infrastructures régionales au bénéfice du monde rural qui l'entoure. On peut difficilement reprocher aux élus locaux de défendre les intérêts de leurs citoyens plutôt que de chercher à plaire à l'ensemble de la population régionale. Il faut donc se poser la question suivante : quelles sont les vraies motivations des élus locaux au sein d'une entité régionale? Est-ce que les maires peuvent pleinement se vouer à l'émancipation régionale en étant d'abord au service de leurs citoyens locaux ? De nombreux exemples nous permettent d'affirmer qu'il est rare que les élus responsables du développement régional jouent leur rôle adéquatement. Il est très difficile en effet d'organiser des services sur une base régionale si toutes les municipalités ne partagent pas les mêmes intérêts ou si les élus municipaux d'une même MRC ne souhaitent pas offrir les mêmes services à leur population. À cet égard, nous avons connu, avant la création des CLD (Centres locaux de développement), une période où le service de développement économique n'était pas offert à l'ensemble des municipalités. Il peut être extrêmement difficile d'organiser du transport collectif à l'échelle d'une MRC si toutes les municipalités ne participent pas à son financement. En conséquence, il est très difficile pour les élus municipaux de jouer un rôle régional quand la volonté générale va à l'encontre de la volonté légitime de leurs conseils municipaux. Les MRC sont généralement des lieux de consensus et, pour cette raison, il peut être difficile de concrétiser certains projets de développement pourtant porteurs d'avenir et de changements.

Si l'élu local siégeant à la MRC peut avoir de la difficulté à bien assumer son mandat régional, comment ce même élu peut-il effectuer efficacement et en toute indépendance son travail au sein des Conférences ré- gionales des élus dont le champ d'action touche l'ensemble d'une région administrative ? Les élus régionaux ne bénéficient pas de conditions optimales pour étudier leurs dossiers supra-municipaux. Ainsi, comment peut-on améliorer les organisations municipales pour qu'elles puissent travailler efficacement au développement du territoire rural et ainsi, participer de façon plus optimale à l'occupation du territoire ? Nous pensons qu'une partie de la solution consiste à renforcer les municipalités et à implanter les conditions propices à un vrai développement régional.

\section{Il est très difficile d'organiser des services sur une base régionale si toutes les municipalités ne partagent pas les mêmes intérêts ou si les élus municipaux d'une même MRC ne souhaitent pas offrir les mêmes services à leur population.}

Le Québec a connu une importante vague de fusions municipales en 2002 avec les résultats que nous connaissons. Le dossier des fusions municipales est devenu un dossier politique pratiquement tabou en raison notamment de l'extraordinaire fiasco des défusions dans la région de Montréal. Pourtant, nous recevons aujourd'hui assez peu d'échos négatifs relativement aux fusions réalisées en milieu rural, et il nous apparaît évident que le pari risqué du gouvernement provincial de l'époque a été relevé. À nos yeux, cet exercice n'a pas été mené aussi loin qu'il aurait dû l'être afin de générer pleinement les retombées positives attendues. Nous pensons que les fusions municipales en milieu rural devraient faire l'objet d'un nouveau débat public, même s'il faut admettre que la tâche serait colossale et les détracteurs nombreux.

Donc, afin de renforcer les municipalités, il nous apparaît très opportun d'étudier collectivement la possibilité de revoir une fois de plus le découpage territorial des institutions municipales rurales. Nous pensons que toutes les municipalités bicéphales de type village/paroisse devraient être fusionnées afin de créer à cette échelle une première cohésion entre le milieu proprement rural et le centre villageois. Nous pensons ensuite que les municipalités de petite taille devraient être fusionnées à leurs voisines afin de stimuler la démocratie et de créer des institutions avec plus de ressources et générant des économies d'échelle. Le tableau 1 présente la relation directe entre la taille des municipalités et le pourcentage d'élus sans opposi- 
tion. Cela démontre clairement que les fusions municipales en milieu rural permettraient d'améliorer la démocratie municipale qui laisse actuellement à désirer.

Une fois l'institution municipale renforcée, voici quelques réflexions sur les actions à poser afin d'améliorer les conditions propices à un bon développement régional. Instaurée en 2001, l'élection facultative du préfet au suffrage universel est un pas dans la bonne direction afin de favoriser un véritable esprit régionaliste au sein des MRC. Par ailleurs, le préfet aura toujours à relever le grand défi qui consiste à rallier les élus locaux à la cause régionale, même s'il peut compter sur l'appui des citoyens habitant l'ensemble du territoire d'une MRC. Pour favoriser encore plus la régionalisation, le Québec pourrait envisager la création de villes-MRC en fusionnant toutes les municipalités à une certaine échelle. À cet égard, le découpage territorial actuel des MRC pourrait servir de base d'étude, mais il pourrait être opportun d'étudier de nouvelles frontières qui permettraient d'équilibrer la masse citoyenne provenant de la campagne et des noyaux urbains tout en conciliant les affinités historiques de différentes communautés. De cette façon, les élus de ces nouvelles villes posséderaient plus d'outils pour agir dans le milieu tout en ayant une vision nécessairement régionale et au service de la ruralité du fait du mandat qu'ils obtiendraient de la population occupant un territoire plus vaste et hétérogène.

\section{Tableau 1 - Répartition des maires élus sans opposition et postes à pourvoir selon la taille des municipalités ${ }^{2}$}

\begin{tabular}{lcccc}
\hline Population & $\begin{array}{c}\text { Nombre total } \\
\text { de postes }\end{array}$ & $\begin{array}{c}\text { Postes à } \\
\text { pourvoir }\end{array}$ & $\begin{array}{c}\text { Élus sans } \\
\text { opposition }\end{array}$ & $\begin{array}{c}\text { Pourcentage d'élus } \\
\text { sans opposition }\end{array}$ \\
\hline 0 à 1999 & 733 & 293 & 440 & $60,0 \%$ \\
2000 à 4999 & 214 & 121 & 93 & $43,5 \%$ \\
5000 à 9999 & 72 & 45 & 27 & $37,5 \%$ \\
10000 à 49999 & 69 & 51 & 18 & $26,1 \%$ \\
50000 à 99999 & 9 & 6 & 3 & $33,3 \%$ \\
100000 et plus & 9 & 9 & 0 & $0,0 \%$
\end{tabular}

À notre avis, il peut exister une autre façon de favoriser une réelle régionalisation: élire des conseillers régionaux plutôt que de confier la gestion des MRC aux élus municipaux. Les maires au sein d'une MRC ont d'abord été élus pour défendre les intérêts de leurs concitoyens municipaux. Cette seule réalité est suffisante pour expliquer d'après nous une bonne partie du manque de cohésion régionale dans les MRC. Il existe des dossiers que les élus locaux ne peuvent pas appuyer régionalement, même si leurs avantages sont assez manifestes à cette échelle. Le maire étant imputable à sa population, très rares sont ceux qui se sentent investis d'une mission régionale qui leur est confiée par leur seule adhésion obligatoire à la structure régionale.

Le travail des élus au sein des MRC est mal payé et très exigeant. Les réunions sont nombreuses et s'additionnent à toutes celles exigées par l'administration municipale locale. Il est évident que les conditions de travail des élus ruraux sont extrêmement difficiles et il est toujours malheureux de constater jusqu'à quel point la population ne reconnaît pas à sa juste valeur le travail des élus. L'élection de conseillers régionaux pourrait permettre de mieux payer les élus, de favoriser des candidatures plus nombreuses et de créer un intérêt pour ces postes d'élus, laissant la voie libre à une meilleure participation élective. Par ailleurs, il n'est pas impossible que cette formule favorise des conflits entre les municipalités et la MRC, ce qui constitue une faiblesse majeure.

Ainsi, dans le contexte actuel, nous pensons que le gouvernement a surestimé la capacité des MRC à s'acquitter de certains mandats à saveur régionale. Avec l'adoption des politiques reliées aux schémas de couverture de risques, aux plans de gestion de matières résiduelles et autres politiques du genre, le gouvernement poursuit généralement l'objectif de favoriser une optimisation des ressources dans un esprit régio- 
nal. À la sortie de ces exercices de concertation, trop peu de MRC ont réussi à remplir le mandat que le gouvernement leur a confié. Il serait extrêmement intéressant que des chercheurs analysent le résultat de ces grands chantiers afin de mettre en relief le problème de volonté politique régionale.

\section{Des pistes de solutions}

Considérant ce que nous avons exposé précédemment, voici quelques pistes de solutions que nous soumettons au lecteur.

Procéder à une réorganisation municipale importante. Afin d'optimiser la planification territoriale, il est impératif de revoir les gouvernements municipaux. Le regroupement des municipalités dans les MRC est un échec aux fins de réaliser une bonne planification territoriale. Une fusion massive des municipalités, pour former des institutions plus fortes et peut-être même des villes-MRC, permettrait d'établir les conditions propices à une vraie planification régionale et à optimiser les organisations municipales tout en leur donnant des moyens plus étendus pour favoriser leur développement.

Favoriser un meilleur aménagement du territoire. Il faut donner une plus grande place à l'aménagement du territoire dans un esprit de complémentarité avec les pôles urbains et poursuivant l'objectif de rationaliser l'occupation du territoire. Comment expliquer qu'après 25 ans d'existence, seulement 61 des 101 MRC du Québec ont un schéma d'aménagement révisé en vigueur ${ }^{3}$ ? L'aménagement du territoire est trop peu souvent une priorité au sein des MRC qui ont pourtant été créées à cette fin. Il faut que le ministère des Affaires municipales et des Régions exige des MRC qu'elles adoptent leurs schémas d'aménagement en imposant des contraintes si elles ne le font pas au-delà d'un certain délai. Il faudrait aussi qu'à défaut d'être constituées en ville-MRC, les MRC soient composées d'élus régionaux indépendants des élus locaux. Les maires ne peuvent efficacement tendre vers une bonne planification régionale compte tenu de leur mandat local.

Une fusion massive des municipalités permettrait d'établir les conditions propices à une vraie planification régionale.
Favoriser la transformation des ressources naturelles et la diversification des économies rurales. Dans l'histoire récente, l'économie de plusieurs milieux ruraux reposait presque exclusivement sur l'exploitation des ressources naturelles. Cette réalité est en pleine mutation pour différentes raisons : effondrement des pêcheries, industrialisation de l'agriculture, réduction de la compétitivité sur les marchés internationaux dans le secteur forestier, etc. Ainsi, le monde rural ne peut plus compter sur la seule exploitation des ressources primaires pour assurer une occupation durable du territoire. Plutôt que d'essayer de perpétuer un modèle d'exploitation familiale des fermes dans le but d'assurer le maintien de la population en milieu rural, ce qui se heurte par ailleurs à des problèmes de productivité et d'accès à une main-d'œuvre spécialisée, nous pensons qu'il serait plus adéquat de chercher à favoriser la deuxième transformation des richesses naturelles directement en milieu rural. Si, collectivement, nous voulons occuper le territoire et maintenir des milieux de vie ruraux propres à l'épanouissement des individus, il faut signer un «autre pacte rural » avec les villes qui ne consiste pas à donner de l'argent aux milieux ruraux, mais plutôt à établir des mécanismes pour garantir l'implantation de certains types d'industries hors des grands centres urbains.

Faire appel à des élus régionaux au mandat élargi. Les conseillers régionaux pourraient prendre sous leur gouverne, en plus des compétences actuellement dévolues aux MRC, des responsabilités inhérentes aux commissions scolaires comme celles relatives au transport ainsi qu'à la gestion des bâtiments et des équipements scolaires. Combinée à l'appropriation du champ de taxation foncier scolaire, nous pourrions dès lors voir apparaître une nouvelle forme de MRC imputable et disposant d'outils structurants. Évidemment, d'autres compétences pourraient leur être confiées tels la justice (Cour municipale), certaines responsabilités du secteur de la santé et les services de police. Des conseillers régionaux seraient mieux placés pour faire les compromis nécessaires qu'exige un réel exercice de planification régionale. D'ailleurs, cela permettrait aux MRC de devenir du même coup un réel enjeu politique. Il n'y a actuellement peu d'élus qui se présentent sur la place publique en période électorale pour débattre des dossiers de MRC. La population n'a donc pas l'occasion d'exprimer son vote à l'égard des dossiers régionaux. Si les MRC étaient confiées à une équipe composée d'un préfet et 
de quelques conseillers, par exemple six comme au sein des conseils municipaux, cela permettrait sans doute aux MRC de mieux répondre à leur mandat.

\section{Des exemples de réussite}

Quoi de mieux que de s'inspirer des réussites pour développer un modèle d'occupation du territoire? Voici deux exemples qui méritent selon nous d'être considérés.

La compagnie Cascades et le développement de Kingsey Falls. La compagnie Cascades est établie à Kingsey Falls depuis 1964 et embauche plus de 14000 personnes à travers le monde. Le siège social de Cascades est encore situé à Kingsey Falls, malgré l'importance de la compagnie. L'unité de recherche et développement est aussi située à cet endroit. Pourtant, Kingsey Falls est une petite municipalité d'un peu plus de 2000 habitants située au cœur d'un milieu rural. Les plus grosses municipalités environnantes sont Victoriaville et Drummondville. Cascades a brillamment remporté son pari d'exploiter une entreprise d'importance dans le milieu rural. Beaucoup de compagnies de cette envergure ont transporté leur siège social dans les métropoles. À contre-courant, Cascades a plutôt investi dans ses ressources humaines et favorise l'épanouissement de ses employés et de leur famille en contribuant au développement de la communauté. Par des mesures financières incitatives, la compagnie a privilégié l'établissement des familles dans la municipalité. Comme il s'agit d'emplois bien rémunérés, l'économie locale s'est diversifiée. La compagnie s'est aussi investie dans la création du parc Marie-Victorin et soutient diverses initiatives locales permettant d'améliorer le milieu de vie. Les retombées de Cascades se font sentir bien au-delà de la seule municipalité de Kingsey Falls. D'autres entreprises pourraient relever ce défi, notamment dans le secteur des technologies de l'information, grâce au déploiement de la fibre optique dans plusieurs milieux ruraux dans les dernières années.

L'École nationale de police de Nicolet. La municipalité de Nicolet profite de l'implantation d'une insti- tution prestigieuse d'enseignement sur son territoire, l'École nationale de police. Située au Centre-du-Québec, cette institution profite à des élèves provenant de partout au Québec. L'institution peut compter sur 275 employés et génère des retombées évidemment très significatives dans le milieu. La ville de Nicolet a su profiter de la présence de l'école en travaillant en partenariat avec celle-ci afin d'offrir des services à la population qu'elle aurait pu difficilement rendre seule. Le rayonnement de l'institution se fait sentir aussi hors du territoire de la ville et contribue de façon appréciable à améliorer la qualité de vie des ruraux.

\section{Conclusion}

Même s'il pouvait être mieux outillé pour efficacement développer son territoire, le milieu rural québécois réussit tout de même à relever certains défis de taille. Malgré la liste des difficultés que vivent les MRC et les municipalités locales, il faut reconnaître que ces organisations réussissent à réaliser des projets majeurs de façon admirable avec des moyens parfois assez limités. Il ne faut pas rejeter tout le modèle actuel.

Par ailleurs, nous espérons que cet article suscitera la réflexion quant à l'avenir des régions rurales, et plus particulièrement sur certaines solutions qui peuvent être envisagées pour mieux outiller le monde municipal pour faire face aux grands défis des prochaines années.

\section{Notes et références}

1 Donald Martel est directeur général de la MRC de NicoletYamaska; Jean-François Albert est directeur général adjoint et aménagiste de la même MRC.

2 MAMR (2005). Profil des élus sans opposition aux élections municipales 2005, Québec, Ministère des Affaires municipales et des Régions.

3 Révision des schémas d'aménagement et de développement, état de situation détaillé - 16 juillet 2007.

http://www.mamr.gouv.qc.ca/amenagement/schemas/amen _amen_revi_rech.asp 